\title{
Statistical approach to evaluate effect of temperature and moisture content on the production of antioxidant naphtho-gamma-pyrones and hydroxycinnamic acids by Aspergillus tubingensis in solid-state fermentation
}

\author{
Quentin Carboué ${ }^{1,2} \cdot$ Catherine Rébufa $^{2} \cdot$ Rayhane Hamrouni $^{2} \cdot$ Sevastianos Roussos $^{2} \cdot$ Isabelle Bombarda $^{2}$
}

Received: 5 May 2020 / Accepted: 20 July 2020 / Published online: 3 September 2020

\begin{abstract}
Non-ochratoxigenic Aspergillus tubingensis G131 is a filamentous fungus that can produce naphtho-gamma-pyrones (N $\gamma \mathrm{Ps})$, polyketide pigments that exhibit interesting antioxidant properties. This study aims to investigate the effect of two critical parameters, temperature and moisture content on the fungus grown in solid-state fermentation using agricultural by-products (vine shoots and wheat bran) as sole medium. From the kinetic productions of secondary metabolites N $\gamma$ Ps (asperpyrone E, dianhydroaurasperone $\mathrm{C}$, fonsecin, fonsecin $\mathrm{B}$ and ustilaginoidin $\mathrm{A}$ ), alkaloids (nigragilin and aspernigrin $\mathrm{A}$ ), degradation products from the solid medium ( $\beta$-D-glucose, $p$-coumaric acid and trans-ethyl ferulate), ergosterol and conidia obtained for different temperatures and moisture contents, a principal component analysis (PCA) was carried out to highlight the production patterns of these compounds. This approach allowed us to determine that fonsecin, the compound of higher interestexhibiting the most interesting antiradical potential—is particularly more produced at $25^{\circ} \mathrm{C}$ and $66 \%$ of moisture content. This study underlines the importance of temperature and moisture content on naphtho-gamma-pyrones and hydroxycinnamic acid production using solid-state fermentation and contributes to the development of agroindustrial by-product valorization.
\end{abstract}

Keywords Solid-state fermentation $\cdot$ Aspergillus tubingensis $\cdot$ Naphtho-gamma-pyrones $\cdot$ Hydroxycinnamic acids . Principal component analysis · Vine shoots

\section{Introduction}

Among the culture methods available, solid-state fermentation (SSF) is a technic that is thousands of years old, first used by mankind for food production but now owning a diversity of applications [1]. It is defined as a microbial culture involving a solid medium in the absence (or near absence) of free water; however, substrate must possess enough moisture to support growth and metabolism of micro-organism [2]. Another important parameter to

Quentin Carboué

quentin.carboue.qc@gmail.com

$\triangle$ Isabelle Bombarda

isabelle.bombarda@univ-amu.fr

1 Vinovalie, ZA Les Portes du Tarn, 81370 Saint-Sulpice-la-Pointe, France

2 Aix Marseille Université, Avignon Université, CNRS, IRD, IMBE, Marseille, France consider is the temperature inside the bioreactor bed. For that matter, heat removal is one of the major constraints in SSF, especially in large-scale processes where metabolic heat is proportionally generated [3]. By the way, heat removal through water evaporation is a possible way to manage the energy inside the bioreactor but requires water replenishment through aeration with vapor saturated air [4].

The influence of moisture content and temperature is essential in the growth and secondary metabolite production of fungi $[5,6]$. However, although some tendencies have been described regarding the effects of moisture content and temperature on secondary metabolism, each fungal strain has its own behavior when facing environmental conditions changes [7, 8]. It is, therefore, important to empirically study those effects when dealing with a strain of industrial interest to optimize the production.

Another essential parameter is the medium composition which has a direct influence on numerous anabolic pathways and, therefore, on the production yields. Besides the 
selection of a proper medium having economic repercussions on the overall process, the valorization of local byproducts as culture medium should be considered to the extent possible $[9,10]$. In the case of southern France, the vine shoots are common by-products and to date, various studies investigated them as a solid medium for the SSF, for the production of feruloyl esterases using Aspergillus terreus and for the production of cellulolytic enzymes by Aspergillus uvarum [11-13].

The black aspergilli (Aspergillus section Nigri) are an important group of species in food mycology, medical mycology and biotechnology [14]. In biotechnology, they are used in many processes for the production of biomass, organic acids, enzymes, and secondary metabolites [15-17]. Aspergillus tubingensis is commonly described as a xerophilic fungus with the optimum growth temperature ranging from 10 to $44{ }^{\circ} \mathrm{C}$ [18]. For that reason, A. tubingensis is a post-harvest mold frequently encountered in grapes where it might cause mycotoxin problems [19]. A. tubingensis strain G131 isolated from grape from a vineyard in Southern France can produce naphtho-gamma-pyrones ( $\mathrm{N} \gamma \mathrm{Ps}$ ) [20]. These secondary metabolites exhibit a broad spectrum of biological activities and particularly radical scavenging activities which depend on the number of hydroxyl groups on the $\mathrm{N} \gamma \mathrm{P}$ skeleton [21-23]. Also, this fungus is non-mycotoxigenic as genome sequencing revealed that the synthetic genes required for the production of ochratoxin A and fumonisin are absent [24]. A study carried out in a liquid medium showed that the production could be improved by decreasing the water activity or by adding an oxidative stress [25].

Overall compounds of interest found in a fermentation extract can be classified into two categories: the compounds directly produced by the fungus-like the secondary metabolites-and the compounds resulting from the fungal enzymatic degradation of the medium-composed by byproducts of vegetal origin. Indeed, plants produce during the phenylpropanoid biosynthesis pathway phenolic hydroxycinnamic acids which have a defensive role as they ensure the transversal junction between polysaccharides-hemicellulose and pectin—and phenolic compounds like lignin [26]. The action of specific fungal enzymes that release them is particularly valuable, e.g., hydroxycinnamic acids $-p$-coumaric acid or trans-ferulic acids - exhibit antioxidant properties [27].

An antioxidant prevents the oxidation of oxidizable matter such as foodstuffs and reduces oxidative stress in living cells, the latter being the physiological consequence of factors such as sunburns, airborne pollutants, or disease [28]. Antioxidant applications, therefore, include various industrial sectors such as health, food, and cosmetics [29]. Moreover, as the demand of products of natural origin increases, microbial non-toxic antioxidant compounds are particularly sought-after for their ability to substitute their synthetic counterparts.

The present study aims to investigate the effect of moisture content and temperature on the $\mathrm{N} \gamma \mathrm{P}$ and hydroxycinnamic acid production by $A$. tubingensis $\mathrm{G} 131$ cultivated under SSF on a medium composed of vine shoots and wheat bran as sole nutrient source. Kinetics of production of various metabolites-including secondary metabolites like $\mathrm{N} \gamma \mathrm{Ps}$ and degradation products - and conidia have been carried out under various levels of moisture contents and temperatures. A statistical approach was used through a principal component analysis (PCA) to visualize rapidly and simultaneously the collected data from all kinetics of production to compare them easily, to highlight eventual production patterns following the various experimental conditions and to interpret the correlations between the different identified compounds to optimize the antioxidant potential of the crude extract. Furthermore, this study contributes to a better knowledge of the growth of A. tubingensis in SSF using byproducts as a culture medium.

\section{Materials and methods}

\section{Fungal strain}

A. tubingensis G131 was provided by École Nationale Supérieure Agronomique de Toulouse (France). The strain was conserved at $4{ }^{\circ} \mathrm{C}$ in a 5 -ml bottle on potato dextrose agar (PDA). The inoculum stock was prepared by propagating the fungus in Erlenmeyer flasks containing PDA. The cultures were incubated at $25^{\circ} \mathrm{C}$ for 7 days. The inoculum suspension was prepared by adding $0.01 \%(\mathrm{v} / \mathrm{v})$ Tween 80 and scraping with a magnetic stirrer to recover the conidia. The number of conidia was counted using a Malassez cell before the inoculation of the solid medium.

\section{Solid-state fermentation (SSF)}

SSF was carried out in duplicate in Erlenmeyer flasks of $250 \mathrm{~mL}$ on $5 \mathrm{~g}$ (dry matter) occupying nearly $40 \%$ of the volume. Particle size ranged from 0.5 to $1 \mathrm{~cm}$. The previously autoclaved solid medium was composed of $50 \%$ vine shoots-acting as a solid support, bringing physical properties like an adapted porosity to the medium - and 50\% wheat bran (w/w) - acting mainly as substrate, bringing nutrients to the fungus-and was inoculated with $2 \times 10^{7}$ conidia $\mathrm{g}^{-1}$ (dry matter) and put in an incubator at various temperatures. The vine shoots were provided by the Laboratoire Européen d'Extraction (La Laupie, France) after they were previously exhausted of their polyphenol content through ethanol extraction to produce value-added compounds [30]. 


\section{Effect of temperature and moisture content}

The temperature effect was investigated at a fixed moisture value of $66 \%$ following four incubation temperatures: 25,30 , 35 , and $40^{\circ} \mathrm{C}$. Moisture effect was studied at a fixed temperature of $25^{\circ} \mathrm{C}$ following three initial moisture values: 50, 60, and $66 \%$. In both cases, production kinetics were established for the following variables: N $\gamma$ Ps including asperpyrone $\mathrm{E}$, dianhydroaurasperone $\mathrm{C}$, fonsecin, fonsecin $\mathrm{B}$ and ustilaginoidin $\mathrm{A}$; some other fungal alkaloids including nigragilin and aspernigrin $\mathrm{A}$; some degradation products from the solid medium tied to the fungal enzymatic activity such as $\beta$-Dglucose, $p$-coumaric acid and trans-ethyl ferulate; a membrane lipid, ergosterol, and the conidia. The cultures were carried out during 14 days and samples have been collected every 2 days.

\section{Ergosterol extraction}

The extraction protocol has been adapted from Saxena et al. (2001) [31]. Briefly, $1 \mathrm{~g}$ of fermented material was introduced in $10 \mathrm{~mL}$ of methanol ( $>99.5 \%$, Sigma-Aldrich, Saint-Louis, USA) solution containing $0.2 \mathrm{M}$ of potassium hydroxide and put in a water bath at $55^{\circ} \mathrm{C}$ for $20 \mathrm{~min}$. The methanol extracts $(5 \mathrm{~mL})$ were collected and stirred with water $(5 \mathrm{~mL})$ and hexane $(10 \mathrm{~mL})$ (Sigma-Aldrich, SaintLouis, USA). After decantation, the hexane phase was removed. The hexane extraction step was carried out two times. The obtained hexane phase was then evaporated under reduced pressure at $30^{\circ} \mathrm{C}$. The dry extract was solubilized in methanol $(2 \mathrm{~mL})$, filtered with a $0.2-\mu \mathrm{m}$ filter (GE Healthcare Systems, Chicago, USA) for subsequent quantification by high-performance liquid chromatography (HPLC) analysis.

\section{Other compound extraction}

The extraction of the investigated compounds ( $\mathrm{N} \gamma \mathrm{Ps}$, degradation products, and other fungal alkaloids) was performed on $1 \mathrm{~g}$ of moist fermented material with analytical ethanol (10 mL) (>99.5\%, Sigma-Aldrich, Saint-Louis, USA) following a self-developed protocol. The mixture was stirred during $1 \mathrm{~h}$. The crude ethanol extract was then filtered with a $0.2-\mu \mathrm{m}$ filter before HPLC analysis.

\section{HPLC analysis}

The HPLC system (Agilent Technologies, Santa Clara, USA) was equipped with a diode array detector (DAD), an RP18 analytical column $(150 \times 5 \mathrm{~mm}, 4.6 \mu \mathrm{m}$ particle size, Zorbax Eclipse XDB, Agilent Technologies, Santa Clara, USA), fitted to an RP18 guard column $(10 \times 4 \mathrm{~mm}$, Agilent Technologies, Santa Clara, USA) and kept at $30{ }^{\circ} \mathrm{C}$ during the analysis. The metabolites were monitored at $280 \mathrm{~nm}$ and the subsequent peaks were observed using ChemStation B.02.01 (Agilent Technologies, Santa Clara, USA).

\section{Ergosterol quantification}

The methanol extracts $(30 \mu \mathrm{L})$ were injected using an autosampler. The isocratic elution was carried out with analytical methanol at a flow rate of $1 \mathrm{~mL} \mathrm{~min}{ }^{-1}$ for $15 \mathrm{~min}$. Ergosterol concentration was determined using a commercial ergosterol standard (Sigma-Aldrich, St. Louis, USA). The results were expressed in mg per $\mathrm{g}$ of dry matter based on the calibration curve obtained from the analysis of standard solutions ranging from 10 to $500 \mathrm{mg} \mathrm{L}^{-1}$.

\section{Other compound quantification}

The crude ethanol extracts $(40 \mu \mathrm{L})$ were injected using an auto-sampler. The mobile phase consisted of acidified water (0.2\% glacial acetic acid) (A) and analytical acetonitrile (B) (Sigma-Aldrich, Saint-Louis, USA). The linear gradient started with $30-100 \%$ of B for 45 min and then $100 \%$ B for

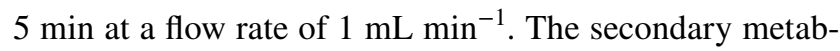
olites investigated being mostly $\mathrm{N} \gamma \mathrm{Ps}$, the concentrations were determined using the commercial standard rubrofusarin A (>98\% purity, Bioviotica Naturstoffe GmbH, Dransfeld, Germany). The results were expressed in mg equivalent rubrofusarin per gram of dry matter based on the calibration curve obtained from the analysis of standard solutions ranging from 2 to $200 \mathrm{mg} \mathrm{L}^{-1}$.

\section{Isolation and radical scavenging assays of the compounds}

Isolation and radical scavenging capacity assays of the compounds were studied in a previous work [23]. Briefly, the radical scavenging capacity assay was carried out as follows: a solution of radical monocation $\mathrm{ABTS}^{\bullet+}$ reacted with $10 \mu \mathrm{mol} \mathrm{L}{ }^{-1}$ of the assayed compound for $5 \mathrm{~min}$, at $25^{\circ} \mathrm{C}$, in 96-well plates. The subsequent decolorization of the monocation in the presence of a radical scavenging compound was measured at $734 \mathrm{~nm}$ using a microplate reader Infinite 200 (Tecan $\odot$, Mennedorf, Switzerland). The results were expressed in Trolox equivalent antioxidant capacity using a calibration curve of 3,4-dihydro-6-hydroxy-2,5,7,8-tetramethyl-2H-1-benzopyran-2-carboxylic acid (Trolox) ranging from 15.625 to $1000 \mu \mathrm{mol} \mathrm{L}^{-1}$. Among the various metabolites that own antioxidant properties, fonsecin and ustilaginoidin A exhibit the highest potentials of $13.32 \pm 0.08$ and $14.59 \pm 1.75$, respectively (not significantly different from the radical scavenging activity of ascorbic acid: $13.23 \pm 0.05)$, when measured with an ABTS assay. This antioxidant potential then decreases as follows: trans-ethyl 
ferulate $(8.32 \pm 0.85), p$-coumaric acid $(6.86 \pm 0.43)$, fonsecin B (5.12 \pm 0.08$)$, dianhydroaurasperone C (4.57 \pm 0.36$)$ and asperpyrone E $(3.29 \pm 0.11)$ [23].

\section{Conidium counting}

$1 \mathrm{~g}$ of each sample was collected, put in distilled water with Tween 80 (Sigma-Aldrich, St. Louis, USA) 0.01\% (v/v), and stirred for $5 \mathrm{~min}$. After appropriate dilution, the conidia were counted using a Malassez cell.

\section{Water content}

The results were expressed in dry weight. To determine the dry weight of a sample, $1 \mathrm{~g}$ of moist fermented material was put in an oven at $105^{\circ} \mathrm{C}$ for $24 \mathrm{~h}$ and was reweighted to measure the percentage of lost water.

\section{Statistical analysis}

Standard deviation was calculated from the duplicate samples and kinetic production curves were made using R software (4.0.0., R Core Team).

PCA was used for reducing the dimensionality of datasets, increasing interpretability but at the same time minimizing information loss. It allowed an interpretable overview of the main information in a multidimensional array [32]. PCA was performed using Unscrambler X software version 10.3 (CAMO, Oslo, Norway).

13 explicative variables were considered: $\beta$-D-glucose, $p$-coumaric acid, trans-ethyl ferulate, nigragilin, aspernigrin $\mathrm{A}$, asperpyrone $\mathrm{E}$, dianhydroaurasperone $\mathrm{C}$, fonsecin, fonsecin B, ustilaginoidin A, ergosterol concentrations and conidia. All were centered, reduced, and divided with the standard deviation to avoid giving importance to high values. The concentration values have not been determined for all moisture contents and temperatures: two matrices were generated. One using the data of kinetics realized under constant moisture content (66\%) at four different temperatures $\left(25,30,35\right.$ and $\left.40{ }^{\circ} \mathrm{C}\right)$ and another with kinetic data with variable moisture contents $(50,60$ and $66 \%)$ at constant temperature $\left(25^{\circ} \mathrm{C}\right)$. The number of principal components (PCs) was determined through a cross-validation. Result interpretation was done from the score plots that allowed explaining the position of samples in the two-dimensional plane generated by the PCs, thanks to certain variables, identified on the plot of correlation loadings. For the analysis related to the temperature effect, 74 samples were used with 962 values for metabolite concentrations and conidium production. Concerning the moisture content effect, 70 samples and 910 concentration values were used.

\section{Results and discussion}

The effects of temperature and moisture content were tested on the growth and metabolite productions within a range of 25 and $40{ }^{\circ} \mathrm{C}$ and 50 and $66 \%$, respectively. Those values were chosen because these ranges were typically used for A. tubingensis optimization and included optimal values for mycotoxins and enzyme production [33-35].

All the tested moisture contents correspond to modalities in which the solid medium is saturated with water, with the presence of a thin aqueous film. Moreover, these moisture contents are constant during the culture process. First of all, we can observe the evolution of the number of conidia and the biomass from the ergosterol production (Fig. 1). Indeed, SSF final product is a fermented matrix for which the separation between the fungus and the culture medium is very challenging, especially when this medium is made of agricultural by-products that are consumed during the fungal growth. Ergosterol is a sterol from the fungal membrane, so it can be used as an indirect biomass indicator [36]. However, it is difficult to convert ergosterol content to a precise biomass value because the ergosterol production depends on the physiological state of the fungus itself depending on many parameters [37]. In this study, two tendencies can be observed: higher temperatures-35 and $40{ }^{\circ} \mathrm{C}$ - seem to favor the fungal growth as the fungus produces more ergosterol and earlier than when the fungus is cultivated under lower temperatures. This production tends to equalize itself from day 7 . This observation is in accordance with the study by Parra and Magan [6] for A. niger which showed that for a fixed water activity value, the growth rate was higher for temperatures of $35,30,25$, and $20^{\circ} \mathrm{C}$, respectively [6]. The moisture content does not lead to major differences in the ergosterol production. Conidia seem to be produced with delay as temperature increases whereas moisture content seems to have a small effect on it.

During the stationary phase, when biomass production decreases, the secondary metabolism starts through endogenous and environmental regulations. Ecologically speaking, this environmental regulation corresponds to a change toward more stressful medium conditions (an oxidative stress, a decline of the carbon source, a temperature rise, etc.) against which the fungus will develop mechanisms of protection through active compound production [38].

The fonsecin and ustilaginoidin A are proportionally produced as moisture content increases and temperature decreases (Figs. 2, 3). This tendency seems to be amplified for the fonsecin production as temperatures of 30,35 and $40{ }^{\circ} \mathrm{C}$ almost completely shut down the production. Although the increasing moisture contents favor fonsecin $\mathrm{B}$, diahydroaurasperone $\mathrm{C}$ and asperpyrone $\mathrm{E}$, temperature 

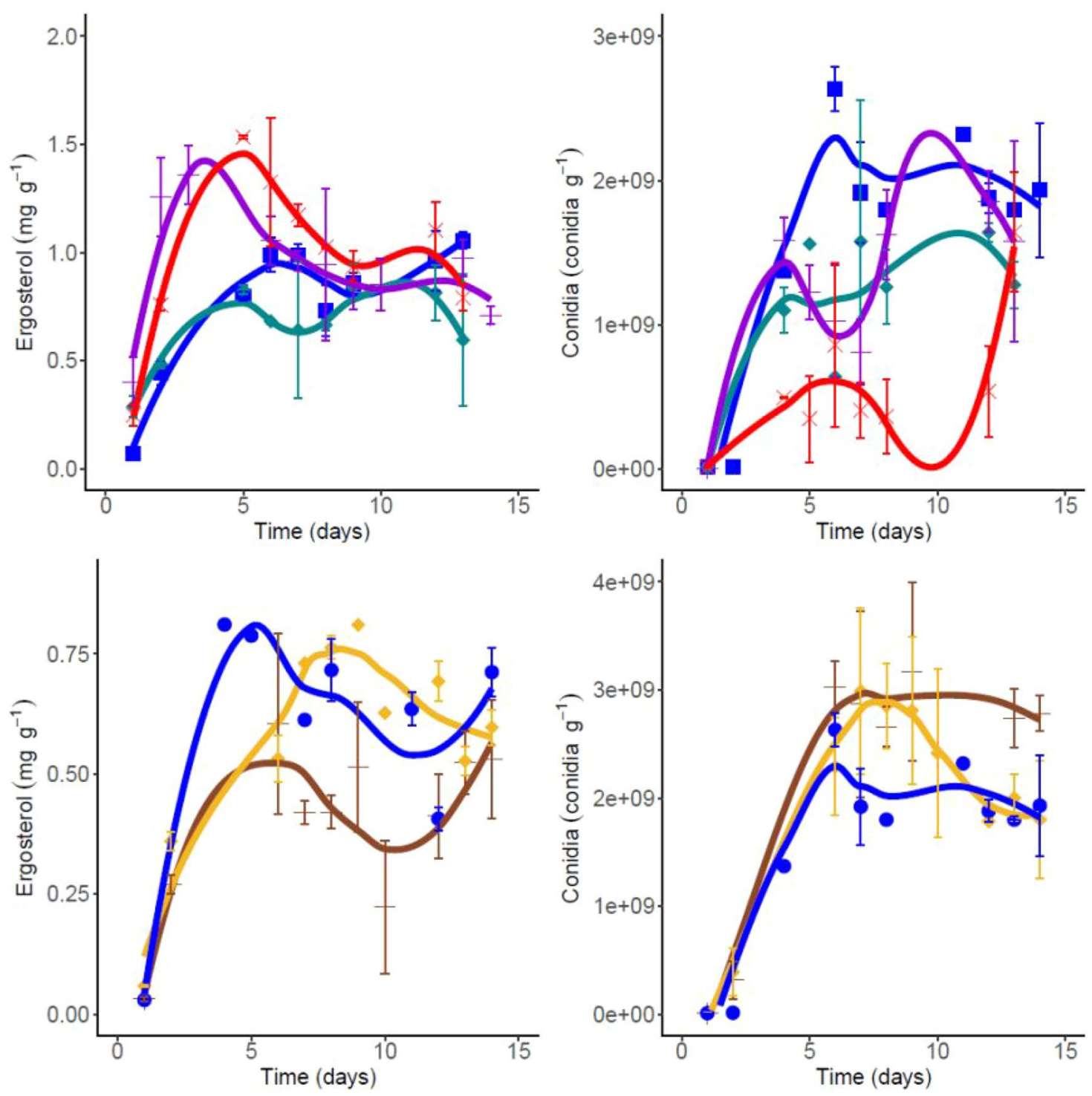

Fig. 1 Fungal growth curves of ergosterol and conidia under various temperatures $\left(\square 25{ }^{\circ} \mathrm{C} \diamond 30{ }^{\circ} \mathrm{C}+35^{\circ} \mathrm{C} \times 40{ }^{\circ} \mathrm{C}\right)$ and moisture contents $(+50 \%-60 \%$ 66\%). Error bars represent the variability associated with the measurements of samples (color figure online)

exhibits different effect on the production as compared with the previous $\mathrm{N} \gamma \mathrm{Ps}$. Indeed, fonsecin $\mathrm{B}$ production is maximized for temperatures of 30 and $35^{\circ} \mathrm{C}$, dianhydroaurasperone $\mathrm{C}$, for $35^{\circ} \mathrm{C}$ and asperpyrone $\mathrm{E}$ for $30^{\circ} \mathrm{C}$.

As for the hydroxycinnamic acids, $p$-coumaric acid and trans-ethyl ferulate are proportionally released into the medium as temperature decreases and as moisture content increases.

Concerning the N $\gamma \mathrm{P}$ yields, a study dealing with the production of $\mathrm{N} \gamma \mathrm{Ps}$ using the same strain of A. tubingensis G131 cultivated in Erlenmeyer flask reported a production of $20 \mathrm{mg} \mathrm{g}^{-1}$ of dry biomass cultivated in liquid conditions using Czapek yeast broth with an osmotic stress-the water activity was set at 0.95 -during 9 days in static conditions [25]. Comparing these results with the one obtained in our study-a total $\mathrm{N} \gamma \mathrm{P}$ production of $0.62 \mathrm{mg} \mathrm{g}^{-1}$ of dry matter obtained after 7 days of growth at $25{ }^{\circ} \mathrm{C}$ and with a moisture content of $66 \%$-is complicated because unlike in SSF although the quantifications of the N $\gamma$ Ps were carried out using the same HPLC technic, the downstream quantification of fungal biomass is easier in liquid systems as it is direct. Using the dry biomass per dry solid medium ratio $\left(0.02 \mathrm{~g} \mathrm{~g}^{-1}\right)$ obtained by Steudler and Bley (2015) through the biomass determination of ergosterol measurement for a strain of Trametes hirsuta grown in SSF on corn silage after 7 days, our $\mathrm{N} \gamma \mathrm{P}$ production yield would be of $31 \mathrm{mg} \mathrm{g}^{-1}$ of dry biomass [39]. This is, however, speculative as there are many different 

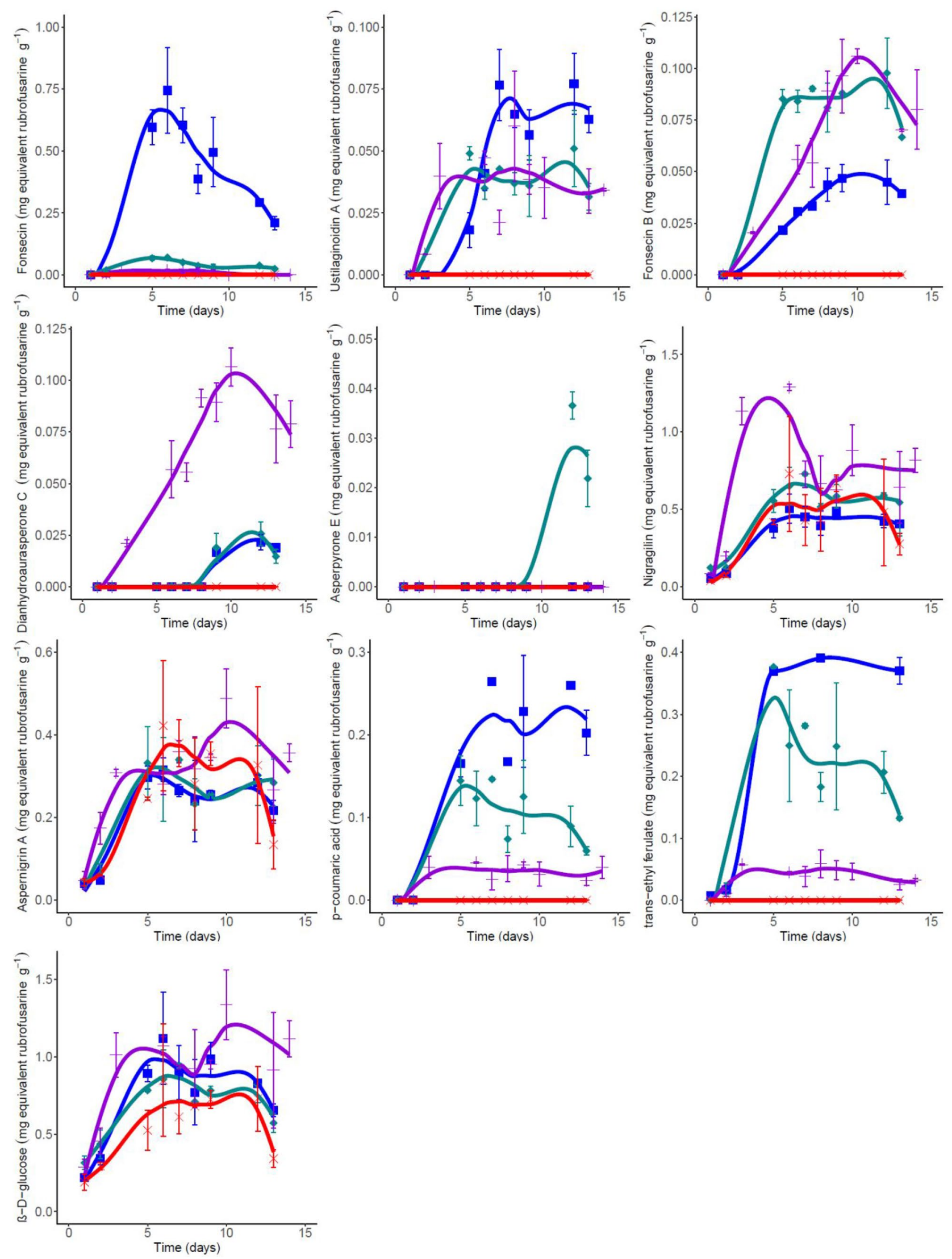
4Fig. 2 Fungal growth kinetic curves of metabolites under various temperatures (square $25^{\circ} \mathrm{C}$ diamond $30{ }^{\circ} \mathrm{C}$ plus symbol $35{ }^{\circ} \mathrm{C}$ multiplication symbol $40{ }^{\circ} \mathrm{C}$ ). Error bars represent the variability associated with the measurements of samples (color figure online)

parameters between the two studies, for example, T. hirsuta is a Basidiomycota whereas A. tubingensis is an Ascomycota. To carry out a proper comparison between the solid and liquid modalities of culture, efficient biomass estimation has to be developed for the solid system. This determination could be carried out by determining the right relation between ergosterol and A. tubingensis G131 biomass production.

As a complement of the observation of the kinetic production curves, another possibility to analyze the data is to use a PCA approach to observe the metabolite behavior under different experimental conditions (kinetics of production related to the temperature and the moisture content variations) and compare them.

In the case of the PCA of the data related to the temperature variations, the cluster of samples in (PC1, PC2) score plot suggests a temporal evolution on $\mathrm{PC} 1$ axis with an explained variance of $46 \%$ (Fig. 4a). This PC1 axis gathers in its negative part the first 2 days of fermentation of the temperature conditions at 25,30 and $35^{\circ} \mathrm{C}$ and the whole kinetic carried out at $40{ }^{\circ} \mathrm{C}$, suggesting a strong negative impact of a temperature of $40^{\circ} \mathrm{C}$ on the metabolism when compared to the others. The PC2 with an explained variance of $23 \%$ shows nonetheless a similar behavior of the data for later times of the kinetics (after day 2) related to 35 and $40{ }^{\circ} \mathrm{C}$ which points are clustered in the positive part of this axis. Points corresponding to higher times of fermentation at $25{ }^{\circ} \mathrm{C}$ follow a distinct pattern as they are located in the negative part of PC2. The points associated with the same time values but with a temperature of $30{ }^{\circ} \mathrm{C}$ exhibit an intermediary behavior as they are placed in the center of PC2. Those various patterns associated with the different conditions are explained by differential production quantities related to each compound.

The interpretation of the correlation loadings allows highlighting the correlations existing between the response variables. Two groups appear: at $25^{\circ} \mathrm{C}$, days $5-13$ are characteristic of a group of molecules comprising fonsecin, ustilaginoidin $\mathrm{A}$ in addition to the hydroxycinnamic acids such as the $p$-coumaric acid and the trans-ethyl ferulate (Fig. 4b). By the way, this result suggests that the fungal $p$-coumaroyl and feruloyl esterases have a temperature optimum of $25^{\circ} \mathrm{C}$. Unlikely, feruloyl esterases of an A. niger strain growing in solid state have shown a temperature optimum for the enzyme activity of $40{ }^{\circ} \mathrm{C}$ [40]. As suggested by the correlation loadings, a second group of compounds which variables are negatively correlated with the previous ones emerges at $35^{\circ} \mathrm{C}$ and after 5 days of fungal growth including aspernigrin $\mathrm{A}$, nigragilin, dianhydroaurasperone $\mathrm{C}$, sugars and to a lesser extent fonsecin B.

A difference of $10{ }^{\circ} \mathrm{C}$ is thus responsible for radical change in the metabolite production.

Concerning data related to the moisture content variation, again the discrimination of samples in (PC1, PC2) score plot suggests a temporal evolution on $\mathrm{PC} 1$ axis with this time an explained variance of $63 \%$ (Fig. 5a).

The PC1 axis gathers in its negative part the points associated with the first days of culture (days 1-4) related to moisture contents of 66 and $60 \%$ and almost all the points of the kinetic (except day 15) related to the moisture content of $50 \%$. The kinetic data obtained for a moisture level of 50 and $60 \%$, for a duration superior to 3 days are, however, more clustered along the PC1 than the data obtained for a moisture level of $66 \%$, indicating that an increase of moisture content is characteristic of a higher metabolic maturity. On the other hand, points associated with the early days of fermentation (until the day 3 ) for the data related to moisture contents of 50 and $60 \%$ are associated in the negative part of PC2 (explained variance of 13\%) with the whole kinetic production point with a moisture content of $66 \%$. Positive part of the PC2 gathers points higher to day 3 of the kinetics assigned to 50 and $60 \%$ of moisture contents.

The observation of correlation loadings shows that most of the N $\gamma$ Ps as well as the hydroxycinnamic acids are produced in higher quantities with moisture content levels of 60 and $66 \%$ for a culture duration ranging between 7 and 15 days. Fonsecin is particularly favored by a moisture content of $66 \%$ (Fig. 5b). Moreover, there is a negative correlation for conidia and aspernigrin productions with the other compounds, suggesting that, for a culture time exceeding 7 days, the production of conidia and aspernigin are favored at a lower moisture content of $50 \%$.

In a strategy of maximization of the antioxidant properties of the crude extract, fonsecin and ustilaginoidin A are the most desirable compounds of the crude extract as they are the most antioxidant compounds [23]. Therefore, to obtain a crude extract with maximum antioxidant properties and because there is a net quantitative difference in the production between fonsecin and the other compounds, fonsecin production must be favored. This pattern of production that maximizes fonsecin production also includes ustilaginoidin A and the hydroxycinnamic acids $p$-coumaric acid and transethyl ferulate.

The $\mathrm{N} \gamma \mathrm{P}$ production process is sensitive to a variation of temperature: this one should be fixed at $25{ }^{\circ} \mathrm{C}$. Indeed, a temperature rise to a value of $35^{\circ} \mathrm{C}$ increases proportionally the production of less hydroxylated $\mathrm{N} \gamma \mathrm{Ps}$ that are, therefore, less antioxidant. Finally, a temperature of $40^{\circ} \mathrm{C}$ disrupts the production. The impact of moisture content on the secondary metabolite production is also significant, in particular, fonsecin production — and to a lesser extent, ustilaginoidin A 

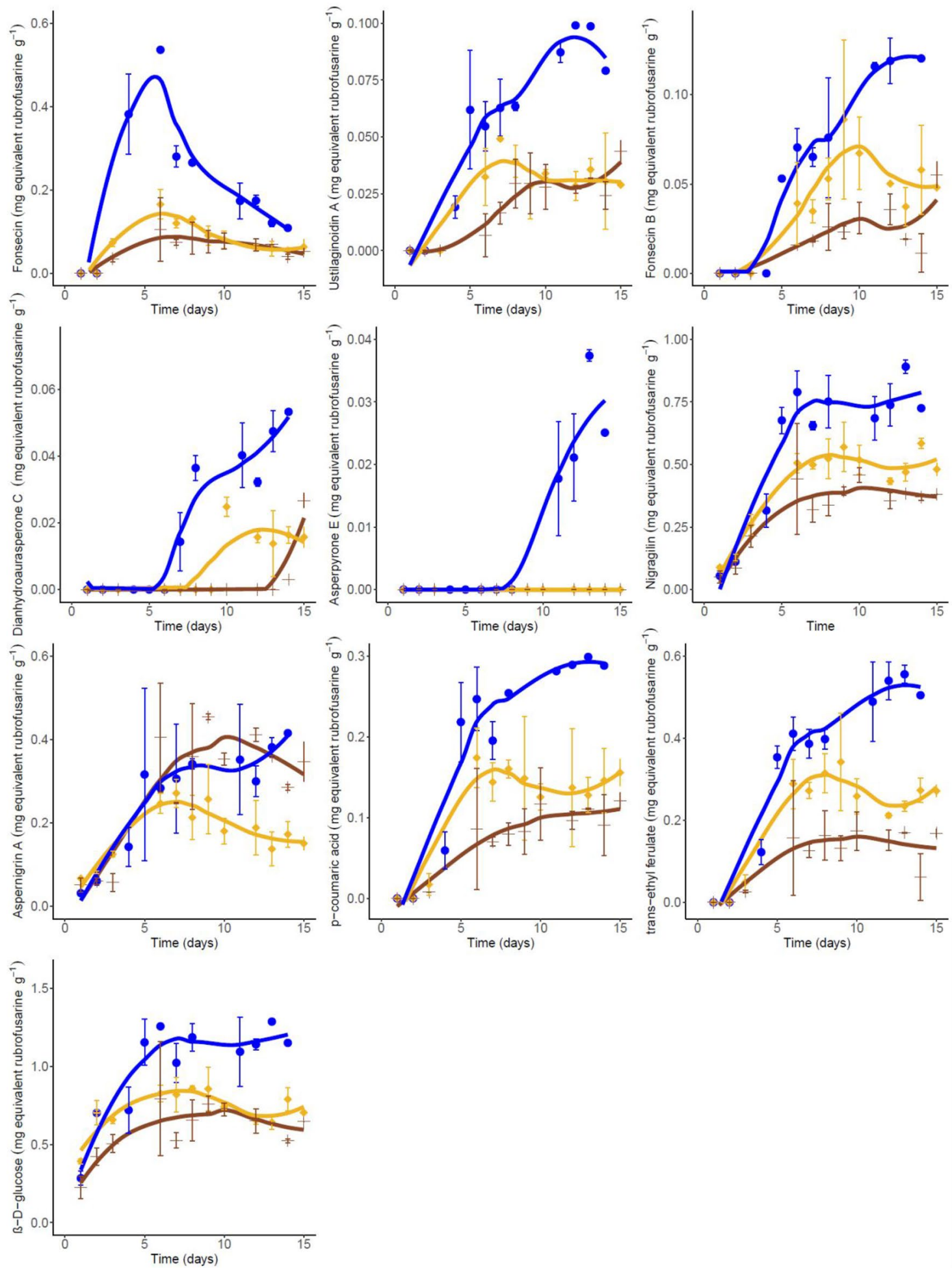

Fig. 3 Fungal growth kinetics curves of metabolites under the variation of moisture content (plus symbol 50\% diamond 60\% circle 66\%). Error bars represent the variability associated with the measurements of samples (color figure online)

and hydroxycinnamic acids. Temperature and moisture content have to be carefully monitored around $25^{\circ} \mathrm{C}$ and $66 \%$, respectively. This affirmation is equally more important if the process is up-scaled. Indeed, the cultures have been performed in flasks at laboratory scale and it is notorious in SSF; an increase of production scale leads to the formation 

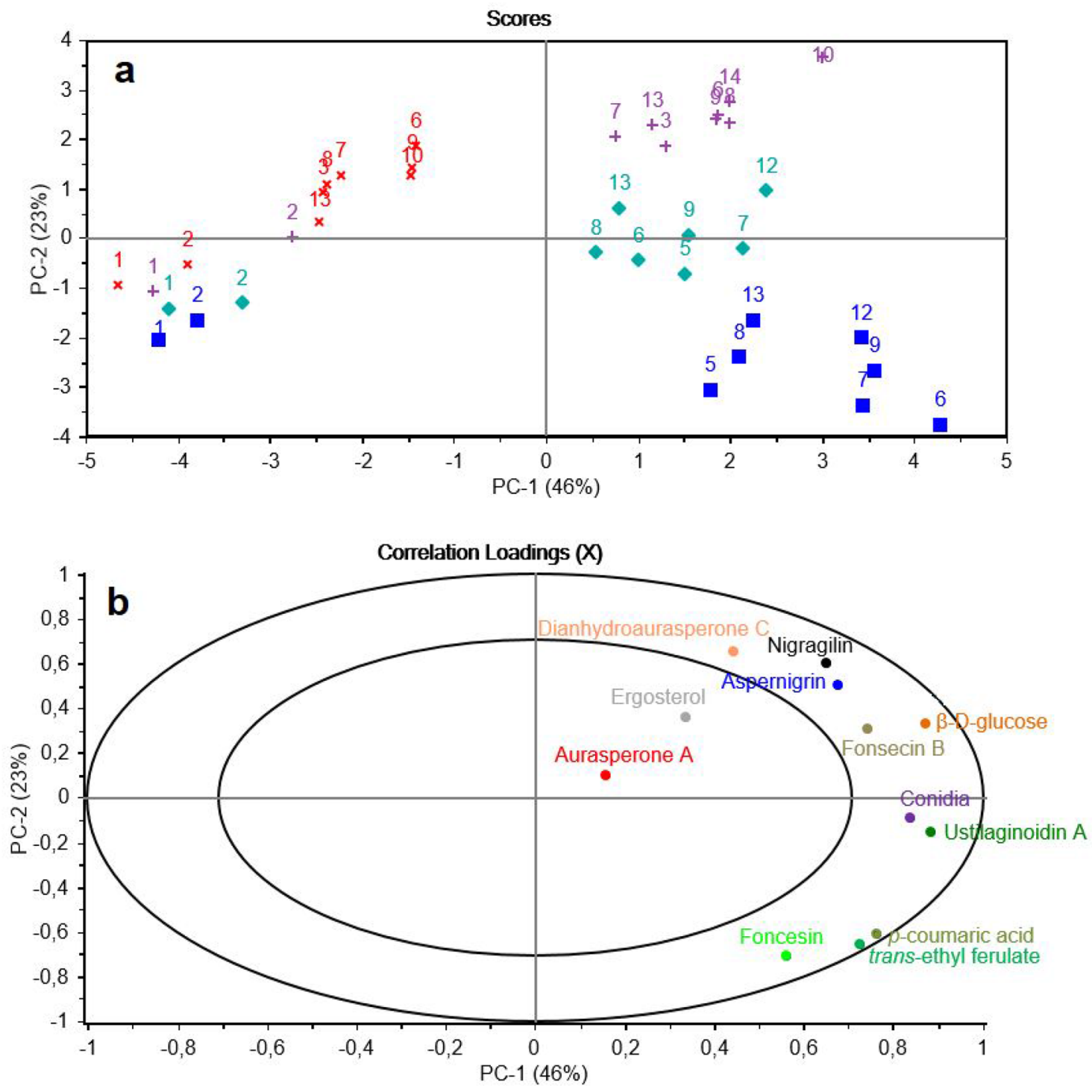

Fig. 4 PCA of the metabolic production related to the temperature variations (square $25{ }^{\circ} \mathrm{C}$ diamond $30{ }^{\circ} \mathrm{C}$ plus symbol $35{ }^{\circ} \mathrm{C}$ multiplication symbol $40^{\circ} \mathrm{C}$ ) in the (PC1, PC2) plane: score plot (a) and correlation loadings (b) (color figure online)

of important thermic and water gradients in the medium bed that can negatively affect the production [41]. However, encouraging results have been obtained at pilot scale for $\mathrm{N} \gamma \mathrm{P}$ production [42].

Also, although the focus is made on the most antioxidant compounds in the crude extract, the complexity of the latter may increase its overall radical scavenging potential through synergistic effects due to other bioactive compounds presence. Further studies have thus to be carried out to determine an eventual synergy. This point supports the fact that the use of the crude extract may be more convenient; also because it would not require implementing an expensive purification process and is notably allowed by the non-mycotoxigenic nature of the fungus. It is also interesting to mention that the culture medium is composed of $50 \%$ of vine shoots and $50 \%$ of wheat bran, making the process based on the valorization of by-products. Concerning vine shoots, it is a local by-product widely produced during the vine pruning in wine-producing French regions. Precisely, the vine shoots used here are previously exhausted of their polyphenol compoundsthe recovered polyphenol are used as value-added molecules-thus making the $\mathrm{N} \gamma \mathrm{P}$ production through SSF, a second way of valorization for this by-product. To complete the circle in a biorefinery approach, it is possible to consider a final way of valorization through composting of the residual fermented matrix to produce fertilizing material for the vine culture. 

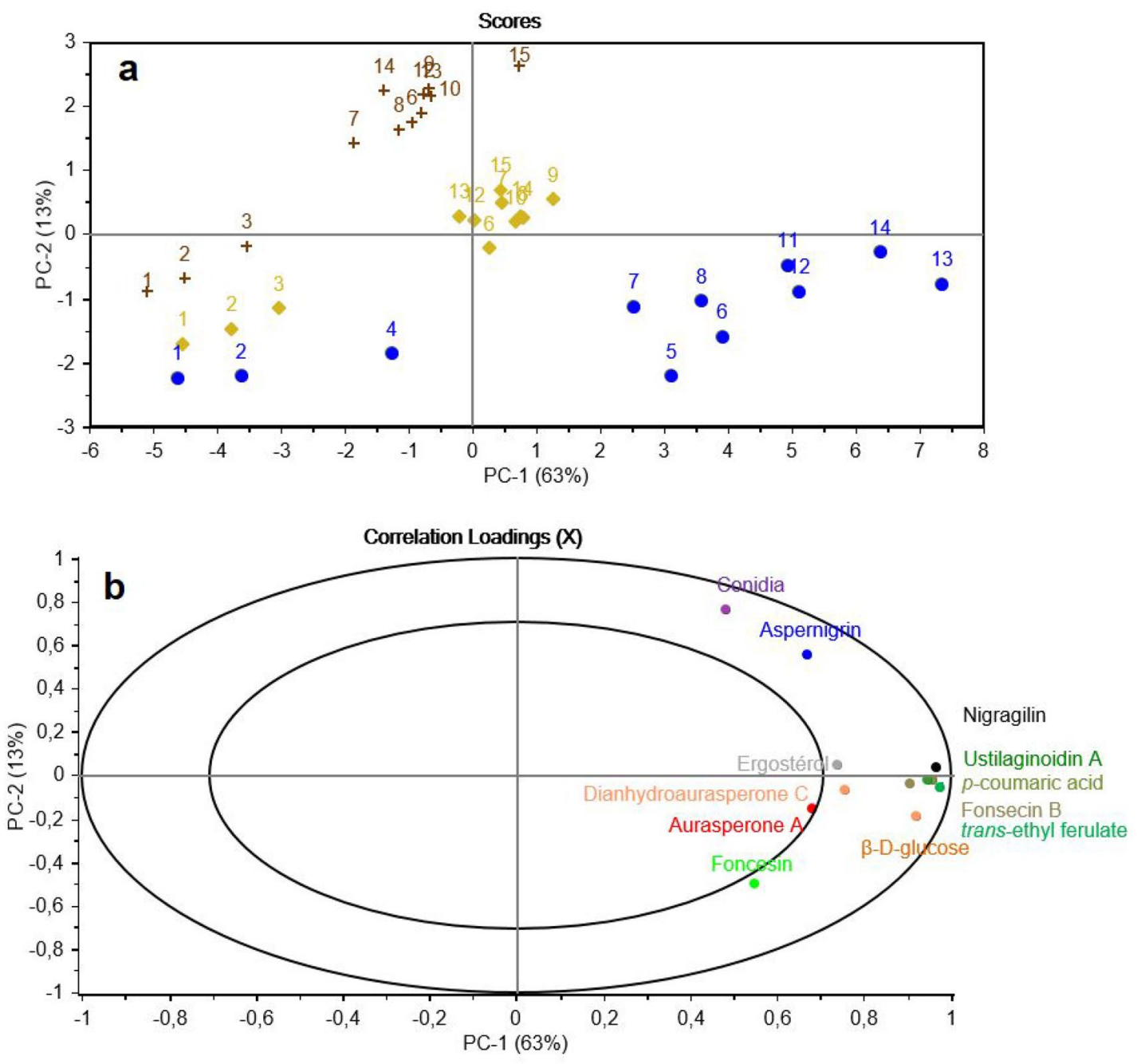

Fig. 5 PCA of the metabolic production related to the moisture content variations (plus 50\% diamond 60\% circle 66\%) in the (PC1, PC2) plane: score plot (a) and correlation loadings (b) (color figure online)

\section{Conclusion}

The PCA approach on the kinetics of fungal compound production under various temperatures and moisture contents rapidly allowed highlighting the patterns of the compound production during the time. Unlike the conidia production which was relatively stable with the various levels of moisture contents and temperatures, the $\mathrm{N} \gamma \mathrm{P}$ production was sensitive to the variation of these parameters. Therefore, although $\mathrm{N} \gamma \mathrm{P}$ production was associated with conidiogenesis on a temporal aspect, it was not correlated to it during the variation of the studied factors. The approach used in this study, growth kinetics under controlled levels of temperature and moisture content, allowed the insight of the metabolic process that was developed during the time. Fonsecin and to a lesser extent ustilaginoidin A and hydroxycinnamic acids are the compounds with higher radical scavenging properties and they follow a similar production pattern depending on temperature and moisture contents changes. In the case of fonsecin, a rise of temperature of $5{ }^{\circ} \mathrm{C}$ over the optimum of $25^{\circ} \mathrm{C}$ drastically diminished the production. This approach also allowed us to determine the optimal temperature and moisture content conditions to maximize the production of antioxidant compounds. Potential applications - for example, included in cosmetic formulations or as a food additive-of the obtained crude extract enriched in non-toxic antioxidant compounds have yet to be investigated.

Acknowledgements Support from Vinovalie (CIFRE ANRT number $2015 / 0027)$ is gratefully acknowledged.

Funding Not applicable.

Data availability All the authors declare that all data and materials as well as software application or custom code support their published claims and comply with field standards. 


\section{Compliance with ethical standards}

Conflict of interest The authors declare that they have no conflict of interest.

\section{References}

1. Soccol CR, da Costa ESF, Letti LAJ et al (2017) Recent developments and innovations in solid state fermentation. Biotechnol Res Innov. https://doi.org/10.1016/j.biori.2017.01.002

2. Pandey A (2003) Solid-state fermentation. Biochem Eng J 13:81-84. https://doi.org/10.1016/S1369-703X(02)00121-3

3. Figueroa-Montero A, Esparza-Isunza T, Saucedo-Castañeda G et al (2011) Improvement of heat removal in solid-state fermentation tray bioreactors by forced air convection. J Chem Technol Biotechnol 86:1321-1331. https://doi.org/10.1002/jctb.2637

4. Singhania RR, Patel AK, Soccol CR, Pandey A (2009) Recent advances in solid-state fermentation. Biochem Eng J 44:13-18. https://doi.org/10.1016/j.bej.2008.10.019

5. Nielsen KF, Holm G, Uttrup LP, Nielsen PA (2004) Mould growth on building materials under low water activities. influence of humidity and temperature on fungal growth and secondary metabolism. Int Biodeterior Biodegrad 54:325-336. https:// doi.org/10.1016/j.ibiod.2004.05.002

6. Parra R, Magan N (2004) Modelling the effect of temperature and water activity on growth of Aspergillus niger strains and applications for food spoilage moulds. J Appl Microbiol 97:429-438. https://doi.org/10.1111/j.1365-2672.2004.02320.x

7. Dhungana B, Ali S, Byamukama E et al (2019) Effects of temperature, water activity, and fungal isolate on ochratoxin A accumulation in oat grain inoculated with Penicillium verrucosum and development of a methodology to screen oat cultivars for ochratoxin A accumulation. Cereal Chem 96:950-957. https ://doi.org/10.1002/cche.10199

8. Pardo E, Marín S, Sanchis V, Ramos AJ (2004) Prediction of fungal growth and ochratoxin A production by Aspergillus ochraceus on irradiated barley grain as influenced by temperature and water activity. Int J Food Microbiol 95:79-88. https:// doi.org/10.1016/j.ijfoodmicro.2004.02.003

9. Abu Yazid N, Barrena R, Komilis D, Sánchez A (2017) Solidstate fermentation as a novel paradigm for organic waste valorization: a review. Sustainability 9:224. https://doi.org/10.3390/ su9020224

10. Hamrouni R, Josiane M, Gregoria M et al (2019) From flasks to single used bioreactor: scale-up of solid state fermentation process for metabolites and conidia production by Trichoderma asperellum. J Environ Manage 252:109496. https://doi.org/10.1016/j. jenvman.2019.109496

11. Pérez-Rodríguez N, Outeiriño D, Torrado Agrasar A, Domínguez JM (2017) Vine trimming shoots as substrate for ferulic acid esterases production. Appl Biochem Biotechnol 181:813-826. https:// doi.org/10.1007/s12010-016-2251-0

12. Pérez-Rodríguez N, Moreira CD, Torrado Agrasar A, Domínguez JM (2016) Feruloyl esterase production by Aspergillus terreus CECT 2808 and subsequent application to enzymatic hydrolysis. Enzyme Microb Technol 91:52-58. https://doi.org/10.1016/j. enzmictec.2016.05.011

13. Salgado JM, Abrunhosa L, Venâncio A et al (2015) Enhancing the bioconversion of winery and olive mill waste mixtures into lignocellulolytic enzymes and animal feed by Aspergillus uvarum using a packed-bed bioreactor. J Agric Food Chem 63:9306-9314. https://doi.org/10.1021/acs.jafc.5b02131
14. Samson RA, Noonim P, Meijer M et al (2007) Diagnostic tools to identify black aspergilli. Stud Mycol 59:129-145. https://doi. org/10.3114/sim.2007.59.13

15. Frisvad JC, Møller LLH, Larsen TO et al (2018) Safety of the fungal workhorses of industrial biotechnology: update on the mycotoxin and secondary metabolite potential of Aspergillus niger, Aspergillus oryzae, and Trichoderma reesei. Appl Microbiol Biotechnol 102:9481-9515. https://doi.org/10.1007/s0025 3-018-9354-1

16. Kang S (2004) Production of cellulases and hemicellulases by Aspergillus niger KK2 from lignocellulosic biomass. Bioresour Technol 91:153-156. https://doi.org/10.1016/S0960 $-8524(03) 00172-\mathrm{X}$

17. Roukas T (2000) Citric and gluconic acid production from fig by Aspergillus niger using solid-state fermentation. J Ind Microbiol Biotechnol 25:298-304. https://doi.org/10.1038/sj.jim.7000101

18. García-Cela E, Crespo-Sempere A, Ramos AJ et al (2014) Ecophysiological characterization of Aspergillus carbonarius, Aspergillus tubingensis and Aspergillus niger isolated from grapes in Spanish vineyards. Int J Food Microbiol 173:89-98. https://doi. org/10.1016/j.ijfoodmicro.2013.12.012

19. Perrone G, Susca A, Cozzi G et al (2007) Biodiversity of Aspergillus species in some important agricultural products. Stud Mycol 59:53-66. https://doi.org/10.3114/sim.2007.59.07

20. Bouras N, Mathieu F, Coppel Y et al (2007) Occurrence of naphtho-gamma-pyrones- and ochratoxin A-producing fungi in French grapes and characterization of new naphtho-gamma-pyrone polyketide (Aurasperone $\mathrm{G}$ ) isolated from Aspergillus niger C-433 ${ }^{1}$. J Agric Food Chem 55:8920-8927. https://doi.org/10.1021/jf071 $406 z$

21. Choque E, El Rayess Y, Raynal J, Mathieu F (2015) Fungal naphtho- $\gamma$-pyrones - secondary metabolites of industrial interest. Appl Microbiol Biotechnol 99:1081-1096. https://doi. org/10.1007/s00253-014-6295-1

22. Xu J (2015) Bioactive natural products derived from mangrove-associated microbes. RSC Adv 5:841-892. https://doi. org/10.1039/C4RA11756E

23. Carboué Q, Maresca M, Herbette G et al (2019) Naphtho-gammapyrones produced by Aspergillus tubingensis G131: new source of natural nontoxic antioxidants. Biomolecules 10:29. https://doi. org/10.3390/biom 10010029

24. Choque E, Klopp C, Valiere S et al (2018) Whole-genome sequencing of Aspergillus tubingensis G131 and overview of its secondary metabolism potential. BMC Genomics 19:200. https:// doi.org/10.1186/s12864-018-4574-4

25. Lopez de Leon L, Caceres I, Bornot J et al (2019) Influence of the culture conditions on the production of NGPs by Aspergillus tubingensis. J Microbiol Biotechnol 29:1412-1423. https://doi. org/10.4014/jmb.1905.05015

26. Dilokpimol A, Mäkelä MR, Aguilar-Pontes MV et al (2016) Diversity of fungal feruloyl esterases: updated phylogenetic classification, properties, and industrial applications. Biotechnol Biofuels 9:231. https://doi.org/10.1186/s13068-016-0651-6

27. Max B, Torrado AM, Moldes AB et al (2009) Ferulic acid and p-coumaric acid solubilization by alkaline hydrolysis of the solid residue obtained after acid prehydrolysis of vine shoot prunings: effect of the hydroxide and pH. Biochem Eng J 43:129-134. https ://doi.org/10.1016/j.bej.2008.09.015

28. Molyneux P (2004) The use of the stable free radical diphenylpicryl-hydrazyl (DPPH) for estimating antioxidant activity. Songklanakarin J Sci Technol 26:212-219

29. Sindhi V, Gupta V, Sharma K et al (2013) Potential applications of antioxidants-a review. J Pharm Res 7:828-835. https://doi. org/10.1016/j.jopr.2013.10.001

30. Carboué Q, Claeys-Bruno M, Bombarda I et al (2018) Experimental design and solid state fermentation: a holistic approach to 
improve cultural medium for the production of fungal secondary metabolites. Chemom Intell Lab Syst 176:101-107. https://doi. org/10.1016/j.chemolab.2018.03.011

31. Saxena J, Munimbazi C, Bullerman LB (2001) Relationship of mould count, ergosterol and ochratoxin A production. Int J Food Microbiol 71:29-34. https://doi.org/10.1016/S0168 $-1605(01) 00584-0$

32. Jolliffe IT, Cadima J (2016) Principal component analysis: a review and recent developments. Philos Transact A Math Phys Eng Sci 374:20150202. https://doi.org/10.1098/rsta.2015.0202

33. Imran M, Hussain A, Anwar Z et al (2019) Beta-glucosidase production optimization from newly isolated Aspergillus tubingensis IMMIS2 using taguchi statistical design. Iran J Sci Technol Trans Sci 43:701-707. https://doi.org/10.1007/s40995-017-0462-z

34. Lahouar A, Marin S, Crespo-Sempere A et al (2017) Influence of temperature, water activity and incubation time on fungal growth and production of ochratoxin A and zearalenone by toxigenic Aspergillus tubingensis and Fusarium incarnatum isolates in sorghum seeds. Int J Food Microbiol 242:53-60. https://doi. org/10.1016/j.ijfoodmicro.2016.11.015

35. Trivedi S, Divecha J, Shah A (2012) Optimization of inulinase production by a newly isolated Aspergillus tubingensis CR16 using low cost substrates. Carbohydr Polym 90:483-490. https:// doi.org/10.1016/j.carbpol.2012.05.068

36. Djajakirana G, Joergensen RG, Meyer B (1996) Ergosterol and microbial biomass relationship in soil. Biol Fertil Soils 22:299_ 304. https://doi.org/10.1007/BF00334573

37. Gessner MO, Chauvet E (1993) Ergosterol-to-biomass conversion factors for aquatic hyphomycetes. Appl Environ Microbiol 59:502-507
38. Keller NP (2019) Fungal secondary metabolism: regulation, function and drug discovery. Nat Rev Microbiol 17:167-180. https:// doi.org/10.1038/s41579-018-0121-1

39. Steudler S, Bley T (2015) Biomass estimation during macro-scale solid-state fermentation of basidiomycetes using established and novel approaches. Bioprocess Biosyst Eng 38:1313-1323. https ://doi.org/10.1007/s00449-015-1372-0

40. Hegde S, Muralikrishna G (2009) Isolation and partial characterization of alkaline feruloyl esterases from Aspergillus niger CFR 1105 grown on wheat bran. World J Microbiol Biotechnol 25:1963-1969. https://doi.org/10.1007/s11274-009-0095-2

41. da Silveira CL, Mazutti MA, Salau NPG (2014) Modeling the microbial growth and temperature profile in a fixed-bed bioreactor. Bioprocess Biosyst Eng 37:1945-1954. https://doi.org/10.1007/ s00449-014-1170-0

42. Carboué Q, Rébufa C, Dupuy N et al (2019) Solid state fermentation pilot-scaled plug flow bioreactor, using partial least square regression to predict the residence time in a semicontinuous process. Biochem Eng J 149:107248. https://doi.org/10.1016/j. bej.2019.107248

Publisher's Note Springer Nature remains neutral with regard to jurisdictional claims in published maps and institutional affiliations. 